\title{
Changes in the Microbiome of Vaginal Fluid after Menopause in Korean Women
}

\author{
Sukyung Kim ${ }^{1}$, Hoonhee Seo ${ }^{1}$, MD Abdur Rahim ${ }^{2}$, Saebim Lee ${ }^{1}$, Yun-Sook Kim ${ }^{3 *}$, and \\ Ho-Yeon Song ${ }^{1,2 *}$
}

${ }^{1}$ Probiotics Microbiome Convergence Center, Soonchunhyang University, Asan, Chungnam 31538, Republic of Korea ${ }^{2}$ Department of Microbiology and Immunology, School of Medicine, Soonchunhyang University, Cheonan, Chungnam 31151, Republic of Korea

${ }^{3}$ Department of Obstetrics and Gynecology, Soonchunhyang University Cheonan Hospital, Chungnam 31151, Republic of Korea

\begin{abstract}
Various microorganisms reside in the human vagina; the vaginal microbiome is closely linked to both vaginal and general health, and for this reason, microbiome studies of the vagina are an area of research. In this study, we analyzed the vaginal microbiome of women before and after menopause to further increase our understanding of the vaginal microbiome and its contribution to general health. We did a 16s rRNA gene-based metagenomic analysis on the vaginal fluids of 11 premenopausal and 19 postmenopausal women in Korea. We confirmed that the taxonomic composition was significantly different between the two groups. In postmenopausal women, species richness was significantly decreased, but species diversity was significantly increased. In particular, among the taxonomic components corresponding to all taxon ranks of the vaginal microbiome, a reduction in Lactobacillus taxa after menopause contributed the most to the difference between the two groups. In addition, we confirmed through metabolic analysis that the lactic-acid concentration was also decreased in the vaginal fluid of women after menopause. Our findings on the correlation between menopause and the microbiome could help diagnose menopause and enhance the prevention and treatment diseases related to menopause.
\end{abstract}

Keywords: Vaginal microflora, premenopausal, postmenopausal, metagenomics, Korean women

Received: June 9, 2021 Accepted:September 1, 2021

First published online: September 3, 2021

*Corresponding authors Y.S. Kim

E-mail: songmic@sch.ac.kr H.Y. Song

E-mail:drsook@schmc.ac.kr, drsook@naver.com

pISSN 1017-7825 elSSN 1738-8872

Copyright $\odot 2021$ by the authors. Licensee KMB. This article is an open access article distributed under the terms and conditions of the Creative Commons Attribution (CC BY) license.

\section{Introduction}

The "microbiome" encompasses all microorganisms and their genomes that inhabit humans, animals, plants, the natural environment, and their surrounding environmental conditions [1]. According to the Human Microbiome Project Consortium report, 4,180 kinds of microorganisms inhabit human feces, and 775, 857, 1,267, and 255 kinds of microorganisms inhabit the buccal mucosa, anterior nares, supragingival plaque, and posterior fornix, respectively [2]. About 38 trillion microorganisms inhabit the average body compared to about 30 trillion cells; therefore, the ratio of the two is close to unity [3]. With this new appreciation of its scale, the importance of the human microbiome is coming into greater focus.

The human microbiome is called our second genome or our other genome because it is closely related to disease and health $[4,5]$. According to MetaHIT (the European Union Project on Metagenomics of the Human Intestinal Tract), the number of genes in the human body is 3,299,822, which is more than 100 times the approximately 23,000 genes that constitute the human genome [6]. These human microbiome genes are classified into 25 groups by functions such as energy metabolism and are connected through a network to perform metabolic functions [6]. For this reason, the human microbiome is associated with numerous diseases, including inflammatory bowel disease, multiple sclerosis, diabetes, allergies, asthma, autism, and cancer, which is why much recent research has been conducted in this regard [7].

Over the years, the female vaginal ecosystem has been formed by co-evolution between a specific microbial partner and a human host [8]. Vaginal secretions contain numerous microbes associated with various diseases, including sexually transmitted infections, bacterial vaginosis, fungal infections, and preterm birth [9], and recently have begun to be analyzed in detail [10]. More specifically, vaginal microbiota analyses of women of childbearing age [11], women with bacterial vaginosis [12], women with pelvic inflammatory disease [13], and of women during pregnancy and the postpartum period, have been conducted [14]. In addition, studies have shown that the microbial environment of the vagina differs from race to race, so studies on the vaginal microbiome need to be carried out with such distinctions in mind [15].

Women typically spend the last third of their lives in menopause following the end of their reproductive years [16]. During menopause, women experience changes in sex hormone levels and various aging-related symptoms 
[16]. Menopause is associated with vasomotor dysfunction, vaginal dryness, mood changes, sleep disturbances, urinary incontinence, cognitive changes, somatic complaints, sexual dysfunction, and poor quality of life, caused by decreased secretion of the ovarian hormones, estrogen and progesterone [17]. Low-dose estrogen therapy has been used for menopausal women, and although such hormone therapy is effective, it has limitations due to side effects, such as mastodynia, fluid retention, nausea, lower extremities cramps, and headaches [18]. Recently, microbiome research has been done on menopausal women to alleviate both diseases caused by menopause and the limitations of existing treatments [19-21].

In this study, we analyze the vaginal microbiome of pre-menopausal and post-menopausal Korean women, and investigate the causes of menopause from a microbiome perspective.

\section{Materials and Methods}

\section{Subjects and Sample Collection}

We enrolled 30 women in the study (18-70 years), including 11 pre-menopausal and 19 post-menopausal women (Table 1). None of the participants had used antibiotics or had had vaginal infections within one month of screening, had not used estrogen products systemically within six months of sampling, and had not used topical estrogen-containing products within one week of sampling. In addition, post-menopausal women had all had amenorrhea for more than 12 months. Women were excluded if they had used douches or lubricants within the prior week. After obtaining informed consent, each participant completed a questionnaire about vaginal or urinary tract symptoms and any history of infection. They self-assessed the severity of several vaginal symptoms, such as vaginal dryness, vaginal irritation or itchiness, pain during urination not associated with infection, vaginal soreness, pain during sexual intercourse, and bleeding after sexual intercourse. The evaluation criteria were classified into mild, moderate, or severe symptoms. After the patients were placed in a lithotomy position on a seismic bed, we inserted a disposable speculum (Taizhou Kangjian Medical Equipment Co., Ltd., China) to collect vaginal secretions using a sterile cotton swab kit and immediately placed the cotton swab into a bucket containing distilled water. After insertion, we closed the lid and moved the bucket to the laboratory. All samples were collected at room temperature, immediately placed in $1 \mathrm{ml}$ of sterile normal saline, then sent to the laboratory within $10 \mathrm{~min}$ for DNA extraction. This study was approved by the Soonchunhyang University Cheonan Hospital Ethics Committee (eIRB) (IRB No. 2019-10-017-005).

\section{Preparation of 16S rRNA Gene Amplicon Libraries, Sequencing}

A diluted saline solution of vaginal secretion was taken and placed in a Lysing Matrix B tube with 0.1-mmdiameter glass beads (MP Biomedicals, USA). We performed bead-beating using a FastPrep-24 5G instrument (MP Biomedicals) for $30 \mathrm{~s}$. We amplified the $\mathrm{V} 4$ region of the $16 \mathrm{~S}$ rRNA gene using primers containing overhang sequences compatible with the Illumina Nextera XT index. The forward primer sequence was 515F (5'TCGTCGGCAGCGTCAGATGTGTATAAGAGACAG-GTGCCAGCMGCCGCGGTAA-3'), and the reverse was 806R (5' GTCTCGTGGGCTCGGAGATGTGTATAAGAGACAG-GGACTACHVGGGTWTCTAAT-3'). We carried out all PCR reactions using a $2 \times$ KAPA HiFi HotStart ReadyMix (Kapa Biosystems, USA). Reactions were run with the following cycling parameters: initial denaturation at $95^{\circ} \mathrm{C}$ for $3 \mathrm{~min} ; 25$ cycles of $95^{\circ} \mathrm{C}$ for $30 \mathrm{~s}$; $55^{\circ} \mathrm{C}$ for $30 \mathrm{~s} ; 72^{\circ} \mathrm{C}$ for $30 \mathrm{~s}$; and a final extension at $72^{\circ} \mathrm{C}$ for $5 \mathrm{~min}$. Subsequently, we performed PCR cleanup.

PCR plates were centrifuged at $1,000 \mathrm{~g}$ for one minute at $20^{\circ} \mathrm{C}$ to collect condensation. We vortexed AMPure XP beads (Beckman Coulter, UK) for $30 \mathrm{~s}$ and added $20 \mu \mathrm{l}$ aliquots to each well of the PCR plate. The entire volume was gently pipetted up and down 10 times, followed by incubation at room temperature for $5 \mathrm{~min}$. The PCR plate was placed on a magnetic stand for $2 \mathrm{~min}$. With the amplicon PCR plate on the magnetic stand, we removed supernatants using a multichannel pipette and washed the beads twice with freshly prepared $80 \%$ ethanol. After allowing beads to air-dry for $10 \mathrm{~min}$, we removed the amplicon PCR plate from the magnetic stand and added $52.5 \mu \mathrm{l}$ of $10 \mathrm{mM}$ Tris ( $\mathrm{pH} 8.5$ ) to each well. After mixing the wells, we incubated the plate at room temperature for $2 \mathrm{~min}$ and placed it on the magnetic stand for $2 \mathrm{~min}$. We transferred $50 \mu \mathrm{l}$ of the supernatant from the amplicon PCR plate to a new 96-well PCR plate.

Following the manufacturer's protocol, the metagenomic library was prepared using a Nextera XT DNA Library Prep Kit (Illumina, USA). After we mixed $5 \mu$ of DNA, Nextera XT Index Primer 1, Nextera XT Index Primer 2, $25 \mu \mathrm{l}$ of $2 \times$ KAPA HiFi HotStart ReadyMix (KAPA Biosystems), and $10 \mu \mathrm{l}$ of PCR Grade water by pipetting, we performed PCR using the following program: initial denaturation at $95^{\circ} \mathrm{C}$ for $30 \mathrm{~s} ; 8$ cycles of $95^{\circ} \mathrm{C}$ for $30 \mathrm{~s} ; 55^{\circ} \mathrm{C}$ for $30 \mathrm{~s}, 72^{\circ} \mathrm{C}$ for $30 \mathrm{~s}$; and a final extension step at $72^{\circ} \mathrm{C}$ for $5 \mathrm{~min}$. Subsequently, we performed PCR cleanup again. The sample was finally diluted twice from $1 \mathrm{nM}$ to $50 \mathrm{pM}$ with $10 \mathrm{mM}$ Tris (pH 8.5). After adding a 10\% PhiX Control library (Illumina), we loaded the library onto an iSeq-100 reagent cartridge (Illumina) and sequenced it on an iSeq-100 (Illumina).

Table 1. Clinical profiles of Korean pre-menopausal $(n=11)$ and post-menopausal women $(n=19)$.

\begin{tabular}{ccc}
\hline Characteristics & Pre-menopausal $(n=11)$ & Post-menopausal $(n=19)$ \\
\hline Age $($ years $)($ mean \pm SD $)$ & $39.4 \pm 2.4$ & $58.5 \pm 3.6$ \\
BMI (mean \pm SD) & $23.2 \pm 3.3$ & $25.9 \pm 3.6$ \\
\hline
\end{tabular}

SD, standard deviation; BMI, Body mass index 
Metagenomics Data Analysis and Statistical Analysis

The FASTQ files obtained by sequencing were subjected to data analysis on the EzBioCloud server (Chunlab, Korea). The raw reads were then pre-processed using Trimmomatic [22] to trim adapters and low-quality bases to produce clean reads. The primers were then trimmed with Myers \& Miller's alignment algorithm [23] at a similarity cutoff of 0.8 . Non-specific amplicons that do not encode $16 \mathrm{~S}$ rRNA were detected by nhmmer in the HMMER software package with $\mathrm{hmm}$ profiles [24]. Unique reads were extracted, and redundant reads were clustered with VSEARCH2's derep_fulllength command [25]. EzBioCloud 16S database [26] was used for a taxonomic assignment using VSEARCH's userarch_global command [25], followed by more precise pairwise alignment in Myers \& Miller's algorithm [23].

Alpha ( $\alpha$ ) diversity indices were analyzed based on ACE (abundance-based coverage estimator) [27], Chao1 [28], Jackknife [29], Shannon/Simpson [30], NPShannon (non-parametric Shannon) [31], and Phylogenetic diversity [32], and beta $(\beta)$ diversity indices were analyzed based on Jenson-Shannon [33], Bray-Curtis [34], Generalized UniFrac [35], and Fast UniFrac [36]. Taxonomic and functional biomarkers were discovered by statistical comparison algorithms of the linear discriminant analysis (LDA) effect size (LEfSe) [37] and KruskalWallis $\mathrm{H}$ tests [38].

\section{Assessment of Organic Acids in Vaginal Samples}

Vaginal swabs were centrifuged at $14,000 \mathrm{~g}$ for $10 \mathrm{~min}$ in a cold room $\left(4-8^{\circ} \mathrm{C}\right)$. Supernatants were transferred to new $1.5 \mathrm{ml}$ microcentrifuge tubes, and methanol (cooled to $-80^{\circ} \mathrm{C}$ ) was added to make a final $80 \%(\mathrm{v} / \mathrm{v})$ methanol solution. We gently shook the solution to mix it and incubated it for $6-8 \mathrm{~h}$ at $-80^{\circ} \mathrm{C}$. Then, it was centrifuged at $14,000 \mathrm{~g}$ for $10 \mathrm{~min}\left(4-8^{\circ} \mathrm{C}\right)$. The supernatant was again transferred to new $1.5 \mathrm{ml}$ microcentrifuge tubes and glass vials for X500B QTOF [39]. Briefly, we performed LC-MS analysis using Exion LC AD coupled with the X500B QTOF system (AB Sciex Pte. Ltd., USA). We injected samples into an ACQUITY UPLC BEH HILIC column (2.1 $\times 50 \mathrm{~mm}, 1.7 \mu \mathrm{m}$, Waters, USA). The mobile phase comprised phase A (water with $10 \mathrm{mM}$ ammonium formate) and phase B (methanol). We performed auto-calibration on every fifth sample. We adapted SCIEX OS software 1.0 to analyze data.

\section{Culture of Lactobacillus from Vaginal Fluid}

The collected vaginal fluid samples were stored at $-80^{\circ} \mathrm{C}$ with $15 \%$ glycerol and used for CFU analysis later. To confirm the Lactobacillus contained in the vaginal fluid of a woman, a culture method using MRS (de Man, Rogosa, and Sharpe) media was performed as in the previous study [40]. On Difco Lactobacillus MRS Agar (288130, BD, USA) plates, $100 \mu \mathrm{l}$ of the collected vaginal sample was spread using a spreader. The plates were then incubated at $37^{\circ} \mathrm{C}$ for 3 days. After incubation, the number of colonies was analyzed.

\section{Results}

Microbial Diversity in Vaginal Metagenomes of Pre-menopausal and Post-menopausal Women

Table 2 and Figs. 1A-1D shows taxonomic composition at the level of phylum, class, order and family for preand post-menopausal women. Analyzing taxonomic classification at the phylum level, it was found that Firmicutes accounted for the highest percentage, followed by Actinobacteria, Proteobacteria, Bacteroides, and Fusobacteria. The proportions for each of the phyla were $77.8 \%, 15.2 \%, 0.0 \%, 2.4 \%$, and $3.7 \%$ in pre-menopausal women, respectively, and $46.1 \%, 24.9 \%, 18.6 \%, 8.0 \%$, and $2.5 \%$ in post-menopausal women, respectively. Firmicutes was significantly reduced $(p=0.012)$, but Proteobacteria $(p=0.0004)$ and Bacteroidetes $(p=0.019)$

Table 2. Distributions of bacterial community structures at different taxonomic levels (phylum, class, order, and family).

\begin{tabular}{|c|c|c|c|c|c|c|c|c|c|c|c|c|c|c|c|}
\hline Phylum & PRE & POST & $\begin{array}{c}p- \\
\text { value }\end{array}$ & Class & PRE & POST & $\begin{array}{c}p- \\
\text { value }\end{array}$ & Order & PRE & POST & $\begin{array}{c}p- \\
\text { value }\end{array}$ & Family & PRE & POST & $\begin{array}{c}p- \\
\text { value }\end{array}$ \\
\hline \multirow[t]{6}{*}{ Firmicutes } & 77.8 & 46.1 & $0.012^{\star}$ & Bacilli & 74.7 & 34.3 & $0.008^{\star \star}$ & Lactobacillales & 74.3 & 31.8 & $0.009^{\star \star}$ & Lactobacillaceae & 65.9 & 24.7 & $0.033^{*}$ \\
\hline & & & & & & & & & & & & Enterococcaceae & 0.0 & 4.6 & 0.846 \\
\hline & & & & & & & & & & & & Streptococcaceae & 8.2 & 5.1 & 0.107 \\
\hline & & & & & & & & Bacillales & 0.0 & 2.4 & $0.021^{*}$ & Bacillaceae & 0.0 & 1.4 & 0.22 \\
\hline & & & & Tissierellia & 1.9 & 6.5 & $0.005^{\star *}$ & Tissierellales & 1.9 & 6.5 & $0.005^{\star \star}$ & Peptoniphilaceae & 1.9 & 6.5 & $0.005^{\star *}$ \\
\hline & & & & Clostridia & 0.0 & 3.0 & $0.013^{\star}$ & Clostridiales & 0.0 & 3.0 & $0.013^{\star}$ & Lachnospiraceae & 0.0 & 2.1 & $0.037^{\star}$ \\
\hline \multirow[t]{3}{*}{ Actinobacteria } & 15.2 & 24.9 & 0.067 & Actinobacteria & 14.4 & 17.9 & & Bifidobacteriales & 13.3 & 15.6 & 0.116 & Bifidobacteriaceae & 13.3 & 15.6 & 0.116 \\
\hline & & & & & & & & Actinomycetales & 0.0 & 1.2 & $0.012^{*}$ & Actinomycetaceae & 0.0 & 1.2 & $0.012^{*}$ \\
\hline & & & & Coriobacte & 0.0 & 6.9 & $0.006^{* *}$ & Coriobacteriales & 0.0 & 6.9 & $0.006^{\star *}$ & Coriobacteriaceae & 0.0 & 6.9 & $0.006^{\star *}$ \\
\hline \multirow[t]{4}{*}{ Proteobacteria } & 0.0 & 18.6 & $0.0004^{* *}$ & $\begin{array}{l}\text { Gammaproteo } \\
\text { bacteria }\end{array}$ & 0.0 & 17.6 & $0.002^{\star *}$ & Pseudomonadales & 0.0 & 6.4 & $0.012^{\star}$ & Pseudomonadaceae & 0.0 & 6.1 & $0.03^{*}$ \\
\hline & & & & & & & & Enterobacterales & 0.0 & 9.8 & $0.004^{* *}$ & Morganellaceae & 0.0 & 4.4 & $0.008^{* *}$ \\
\hline & & & & & & & & & & & & Enterobacteriaceae & 0.0 & 1.9 & $0.016^{*}$ \\
\hline & & & & & & & & Pasteurellales & 0.0 & 1.3 & 0.651 & Pasteurellaceae & 0.0 & 1.3 & 0.651 \\
\hline Fusobacteria & 3.7 & 2.5 & 0.272 & Fusobacteria & 3.7 & 1.5 & 0.272 & Fusobacteriales & 3.7 & 1.5 & 0.272 & Leptotrichiaceae & 3.7 & 1.5 & 0.292 \\
\hline
\end{tabular}

Unit: \%; Those present at less than $1 \%$ are not included. ${ }^{\star} p<0.05 ;{ }^{* *} p<0.01$

PRE, pre-menopause group; POST, post-menopause group. 
A

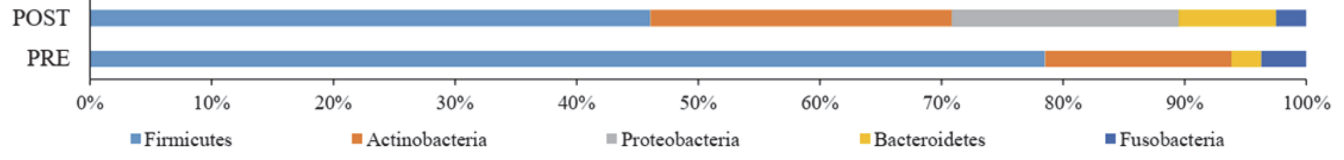

B

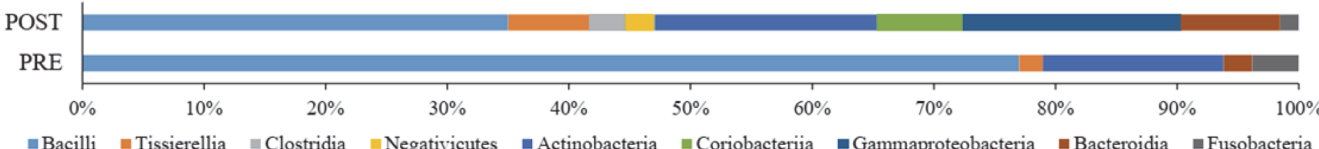

C

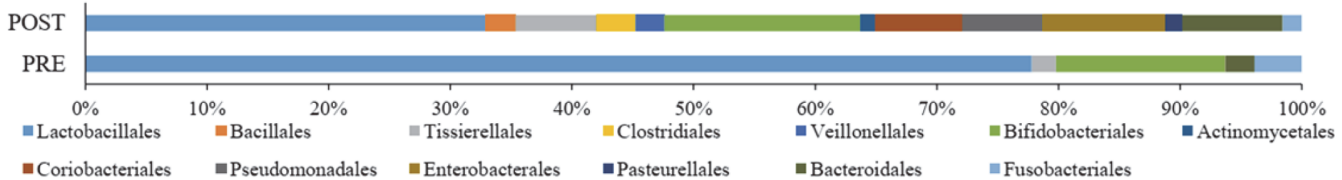

D

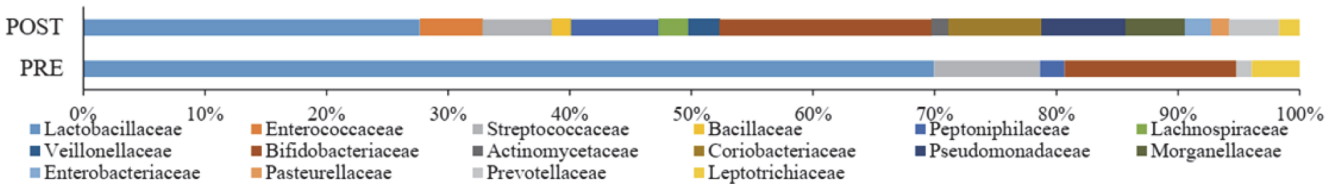

E

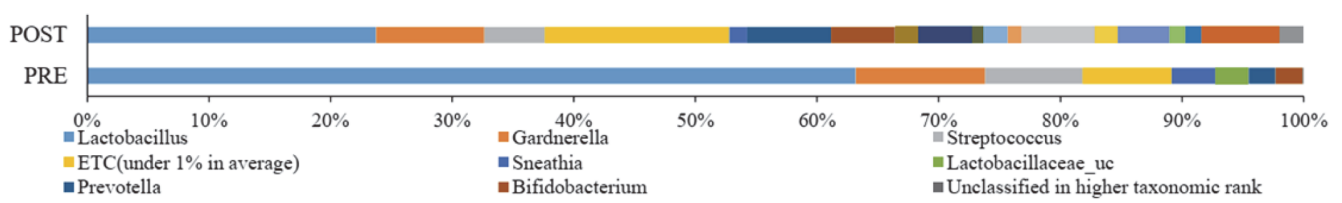

Fig. 1. Average taxonomic compositions of pre-menopausal and post-menopausal women. The pre-menopause group (PRE) and post-menopause group (POST) were further classified at the (A) phylum, (B) class, (C) order, (D) family, and (E) genus. Those with relative abundances less than $1 \%$ were expressed as ETC.

were significantly increased in post-menopausal compared to pre-menopausal women. At the class level, Bacilli, which correspond to the Firmicutes phylum, accounted for the highest proportion and were significantly decreased $(p=0.008)$ in post-menopausal women $(34.3 \%)$ compared to pre-menopausal women $(74.7 \%)$. In contrast, Tissierellia, Clostridia, Negativicutes, Coriobacteriia, and Gammaproteobacteria were significantly increased post-menopausally compared to pre-menopausal $(p=0.005,0.013,0.023,0.006$, and 0.002 , respectively). At a lower taxonomic level, Lactobaciilales order was significantly depleted $(p=0.009)$ in postmenopausal (31.8\%) with respect to pre-menopausal women (74.3\%). On the other hand, Bacillales $(p=0.021)$, Tissierellales $(p=0.005)$, Clostiridiales $(p=0.013)$, Veillonellales $(p=0.023)$, Actinomycetales $(p=0.012)$, Coriobacteriales $(p=0.006)$, Pseudomonadales $(p=0.012)$, and Enterobacterales $(p=0.004)$ were significantly increased post-menopausally. Analyzing the vaginal microbiota composition at the family level, Lactobacillaceae was significantly decreased ( $p=0.033)$ post-menopausally $(24.7 \%$ compared to $65.9 \%)$; whereas Peptoniphilaceae $(p=0.005)$, Veillonellaceae $(p=0.023)$, Actinomycetaceae $(p=0.012)$, Coriobacteriaceae $(p=0.006)$, Pseudomonadaceae $(p=0.03)$, Morganellaceae $(p=0.008)$, Enterobacteriaceae $(p=0.016)$, and Prevotellaceae $(p=$ $0.045)$ showed significant increases in post-menopausal women. At the genus level, Lactobacillus showed significant reductions $(p=0.037)$ post-menopausally $(23.7 \%$ compared to $63.2 \%)$, whereas Prevotella $(p=0.023)$, unclassified Lactobacillaceae ( $p=0.033)$, Escherichia $(p=0.041)$, Pseudomonas $(p=0.03)$, Proteus $(p=0.017)$, Finegoldia ( $p=0.006)$, and Atopobium ( $p=0.006)$ were significantly increased after menopause (Fig. 1E).

\section{Correlation between Pre-Menopause and Post-menopause Groups}

Species richness in the pre-menopause group was significantly higher than in post-menopause (Ace, $p=0.037$; Chao1. $p=0.045$; Jackknife, $p=0.041$; the number of OTUs, $p=0.045$ ) (Fig. 2). In species diversity, the Simpson index was significantly lower (Simpson, $p=0.001$ ) and was significantly higher in the NPShannon, Shannon, and phylogenetic diversity indices (NPShannon, $p=0.009$; Shannon, $p=0.008$; phylogenetic diversity, $p=0.027$ ) in the post-menopause samples (Fig. 3). Principal coordinate analysis (PCoA) plots revealed significant differences between the pre-menopausal and post-menopausal groups based on the Unweighted Pair Group Method using Jensen-Shannon divergence, Bray-Curtis, Generalized UniFrac, and UniFrac (Fig. 4). Unweighted Pair Group 


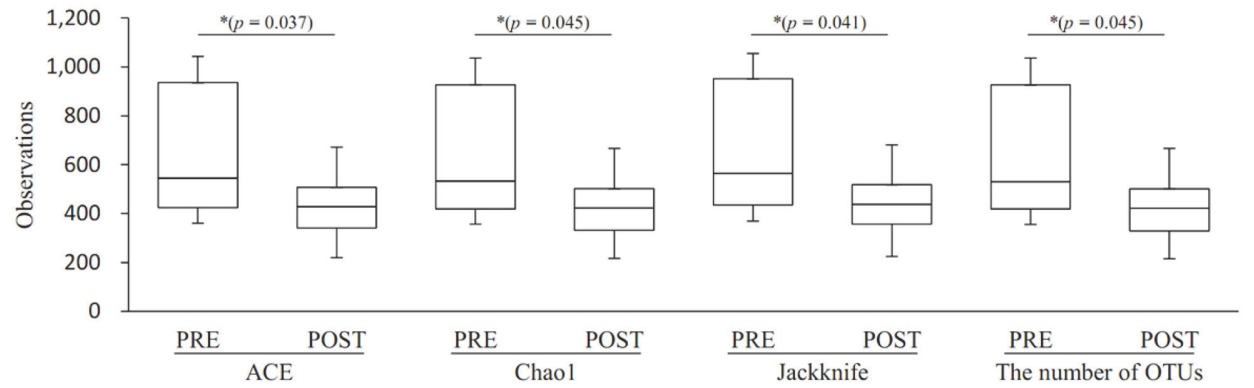

Fig. 2. Boxplot of species richness indices. The species richness of the pre-menopause group (PRE) or post-menopause group (POST) was analyzed by Ace, Chaol, Jackknife, and the number of OTUs method. The horizontal thick black band represents the median value, and the boxplot margins indicate the first and third quartiles.
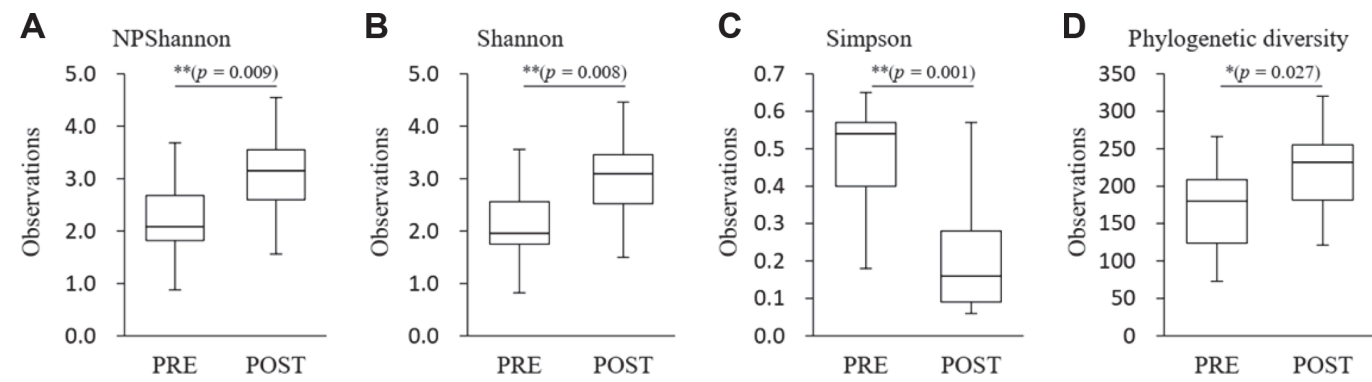

Fig. 3. Boxplot of species diversity indices. The species diversity of the pre-menopause group (PRE) and post-menopause group (POST) was analyzed by (A) NPShannon, (B) Shannon, (C) Simpson $(p=0.007)$, and (D) phylogenetic diversity. The horizontal thick black band represents the median value, and the boxplot margins indicate the first and third quartiles.

A
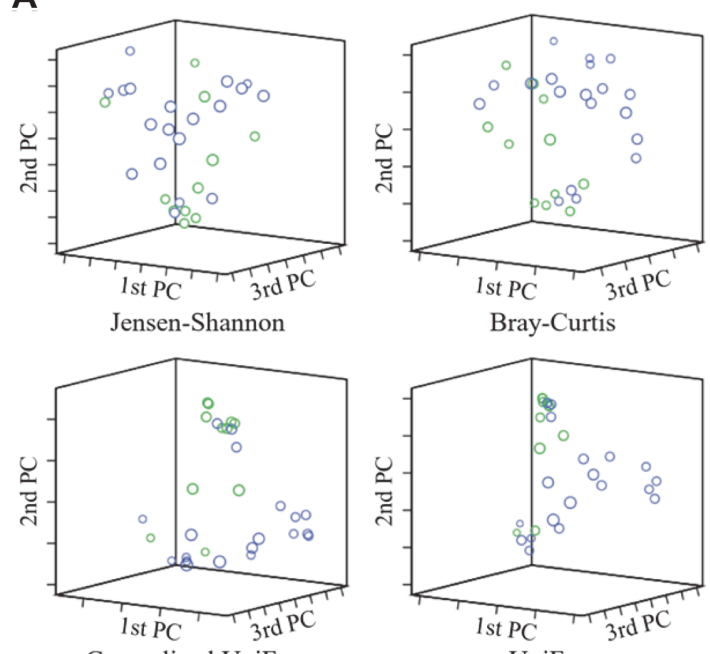

Generalized UniFrac

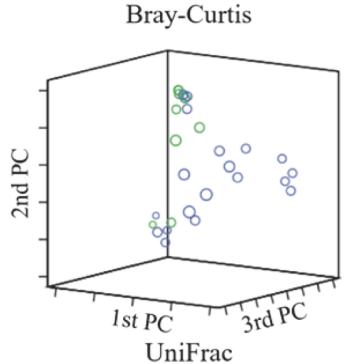

B

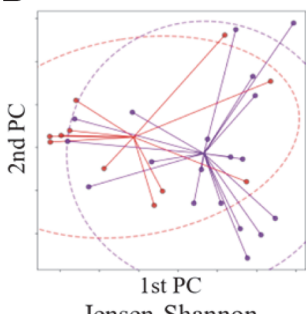

Jensen-Shannon

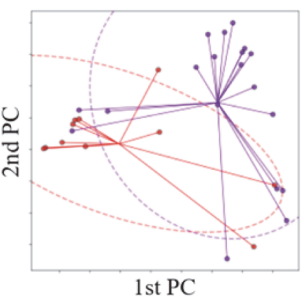

Generalized UniFrac

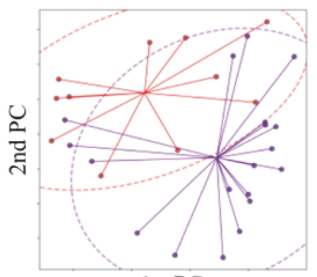

1st PC

Bray-Curtis

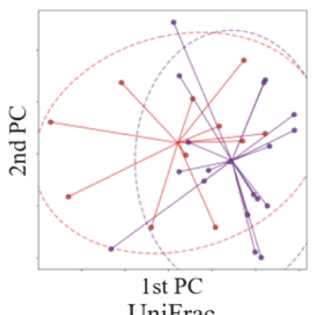

Fig. 4. Principal coordinate analysis (PCoA) of the bacterial communities present in the pre-menopause group (PRE) and post-menopause group (POST). (A) Beta diversity 3D PCoA plot based on Jensen-Shannon divergence, Bray-Curtis, Generalized UniFrac, and UniFrac. (B) Beta diversity PCoA plot with an ellipse at 95\% confidence interval based on Jensen-Shannon divergence, Bray-Curtis, Generalized UniFrac, and UniFrac. The blue/purple color indicates post-menopausal, and the light green/red color indicates pre-menopausal, respectively.

Method with Arithmetic mean (UPGMA) hierarchical clustering was analyzed in Fig. 5. Beta-set-significance analysis showed significant differences in the genus or species level between the pre-menopausal and postmenopausal groups (Table 3). 
A

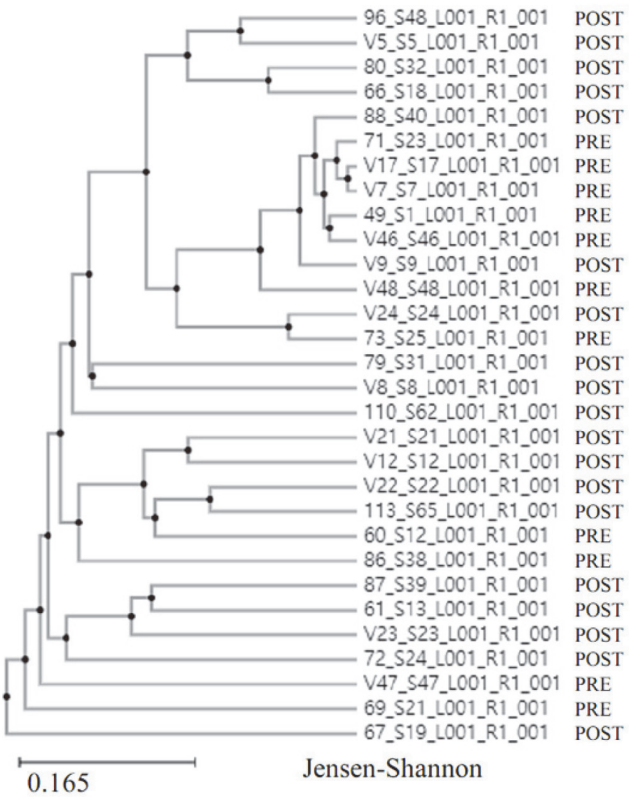

C

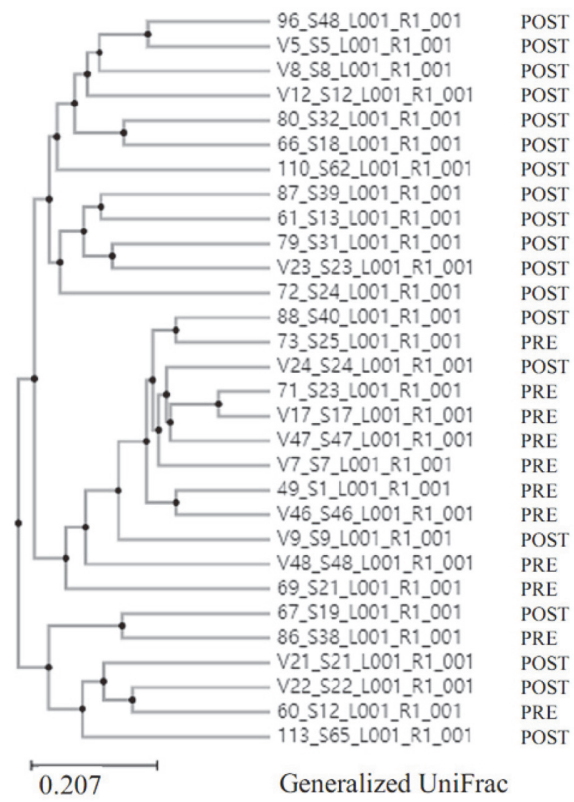

B

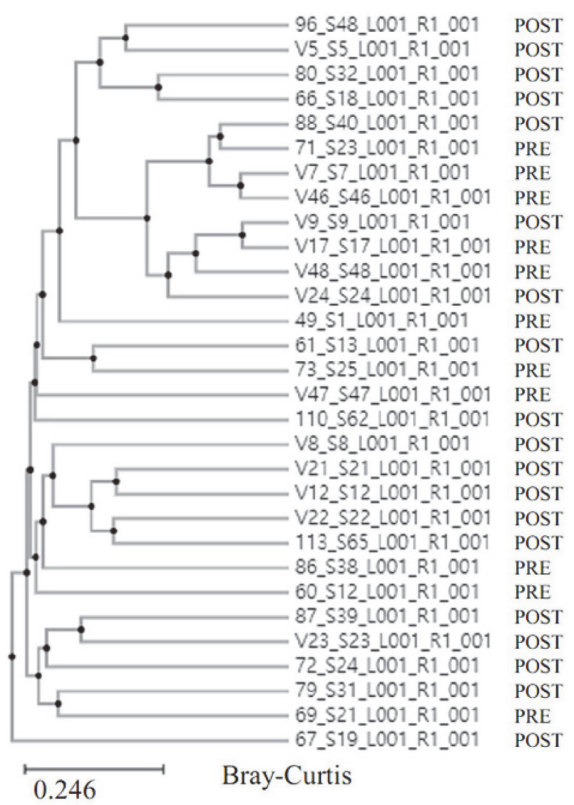

D

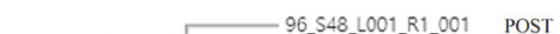
V5_S5_L001_R1_001 POST 80_S32_L001_R1_001 POST V8_S8 L001_R1_001 POST V8_S8_L001_R1_001 POST 110_S62_L001_R1_001 POST

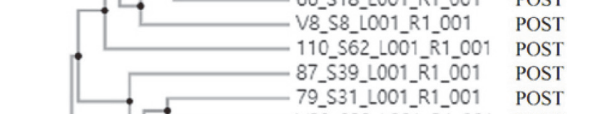

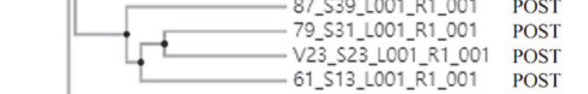
88_S40_L001_R1_001 POST -71_S23_L001_R1_001 PRE - V17 S17 L001 R1 001 PRE $\rightarrow[-V 7571001$ R1 001 PRE - 49 S1 001 R1 001 - V24524 - 73 S25 $001-011$ PRE V47_S47_L001_R1_001 PRE V V SS9_LO01_R1_001 POST - V46_S46_L001_R1_001 PRE V48_S48_L001_R1_001 PRE 69_S21_L001_R1_001 $\quad$ PRE 72_S24_L001_R1_001
POST
67_S19_L001_R1_001 POST

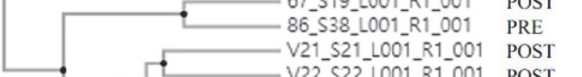
V22_S22_L001_R1_001 POST POST

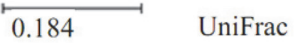

Fig. 5. Clustering using the Unweighted Pair Group Method with Arithmetic mean (UPGMA). Pre-menopause group (PRE) and post-menopause group (POST) were analyzed by (A) Jensen-Shannon, (B) Bray-Curtis, (C) Generalized UniFrac, and (D) UniFrac.

Table 3. Statistical analysis of beta-diversity.

\begin{tabular}{lcc}
\hline Beta diversity distance & Species & Genus \\
\hline Jensen-Shannon & ${ }^{*}(p=0.018)$ & ${ }^{*}(p=0.029)$ \\
Bray-Curtis & ${ }^{*}(p=0.011)$ & ${ }^{* *}(p=0.008)$ \\
Generalized UniFrac & ${ }^{* *}(p=0.002)$ & ${ }^{* *}(p=0.002)$ \\
UniFrac & ${ }^{*}(p=0.004)$ & ${ }^{* *}(p=0.004)$ \\
\hline
\end{tabular}

Permutational multivariate analysis of variance (PERMANOVA) results demonstrated the beta set significance between premenopausal and post-menopausal women. ${ }^{*} p<0.05 ;{ }^{* *} p<0.01$.

\section{Taxonomic Biomarker Discovery}

To identify the specific bacterial taxa associated with menopause, we compared the vaginal microbiota groups using LEfSe in the Galaxy workflow framework with the parameters set at $p<0.05$ and LDA score $=2.0$ (Fig. 6A). 
A

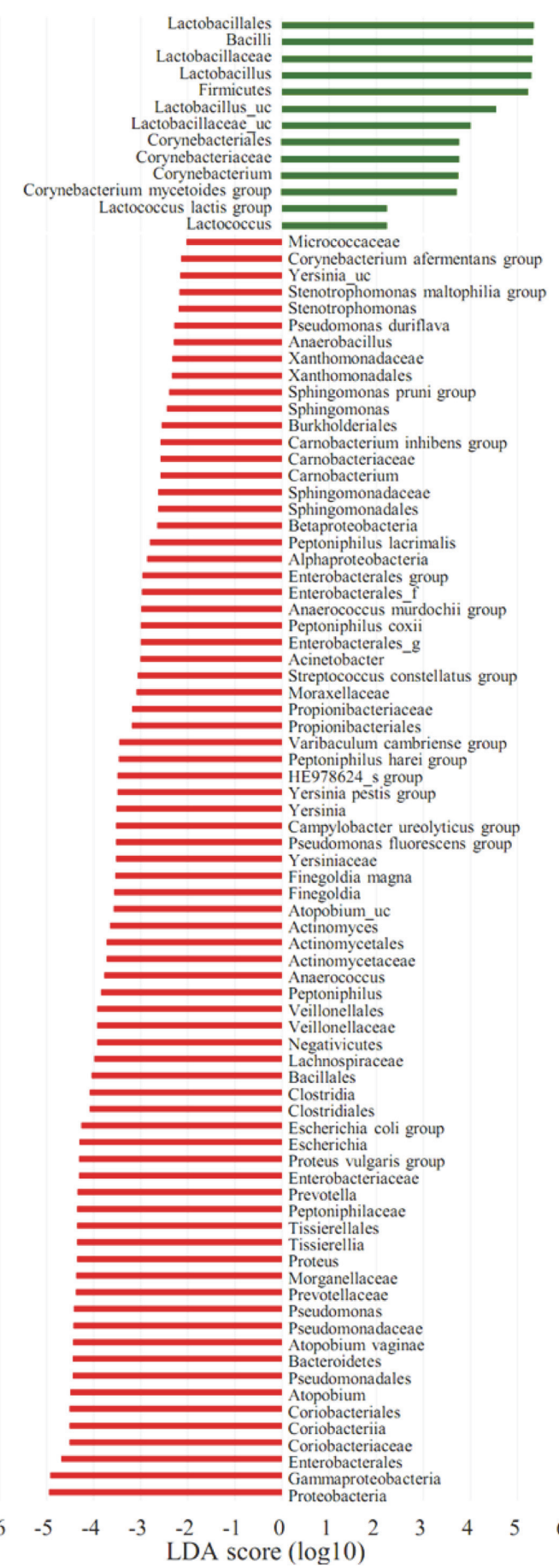

B
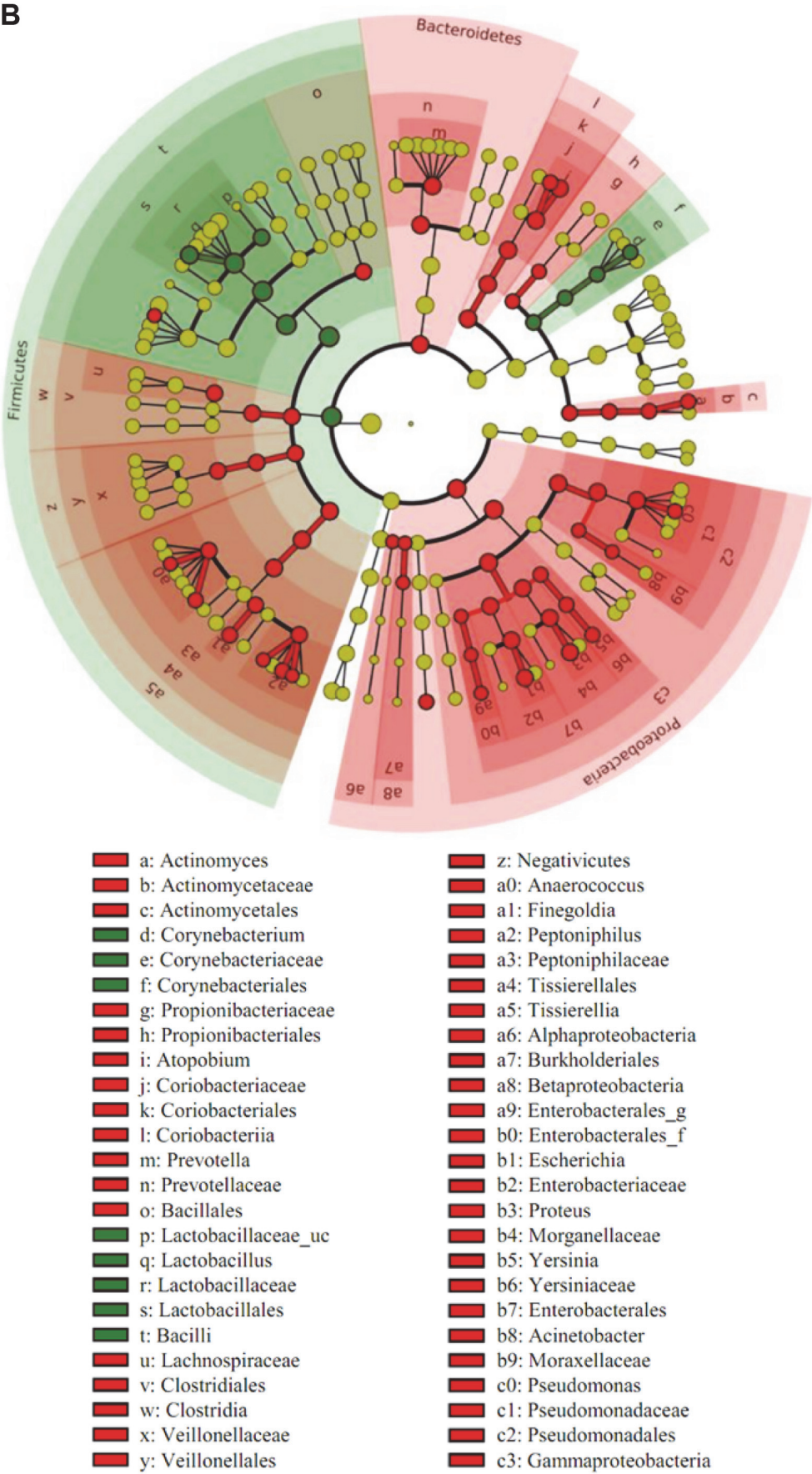

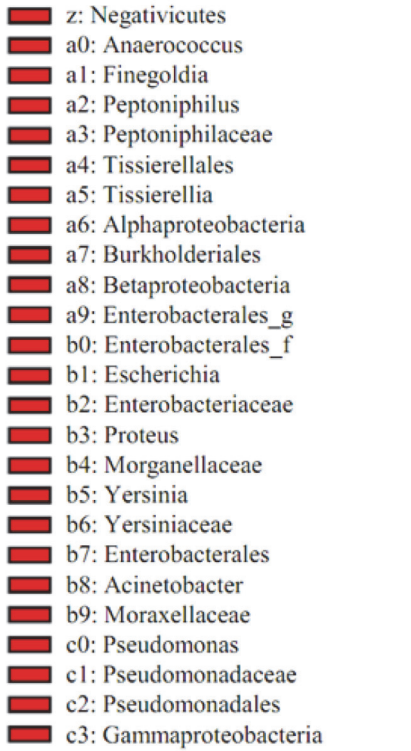

Fig. 6. Distinct taxa identified in the pre-menopause group (PRE) and post-menopause group (POST) using LEfSe (Linear discriminant analysis Effect Size) analysis. (A) Taxonomic cladograms were derived from a LEfSe analysis with linear discriminant analysis (LDA) scores greater than 2 and significance at $\alpha<0.05$, as determined by the Kruskal-Wallis test. (B) Cladogram representing statistically significant differences in bacterial clades between premenopausal and post-menopausal women. Small circles shaded with different colors in the diagram represent the abundances of the taxa in each respective group. Each circle's diameter is proportional to the taxon's abundance. Regions in red indicate taxa enriched in the post-menopause group, while regions in green indicate taxa enriched in the pre-menopause group.

Only those with $p<0.05$ and LDA effect size $>4$ are summarized in Table 4 . Taxonomic cladogram obtained from LEfSe analysis of $16 \mathrm{~S}$ sequences is shown in Fig. 6B. The taxa with an LDA effect size exceeding 5 included Lactobacillales (5.34), Bacilli (5.32), Lactobacillaceae (5.31), Lactobacillus (5.29), and Firmicutes (5.23) (Fig. 7). 
Table 4. Kruskal-Wallis $\mathrm{H}$ tests and LEfSe analysis identification of associations between pre-menopausal and post-menopausal women.

\begin{tabular}{|c|c|c|c|c|c|}
\hline Taxon name & Taxon rank & LDA effect size & $p$-value & $\operatorname{PRE}(\%)$ & POST (\%) \\
\hline Lactobacillales & Order & 5.34 & 0.01 & 74.34 & 31.84 \\
\hline Bacilli & Class & 5.32 & 0.01 & 74.68 & 34.27 \\
\hline Lactobacillaceae & Family & 5.31 & 0.03 & 65.87 & 24.69 \\
\hline Lactobacillus & Genus & 5.29 & 0.04 & 63.16 & 23.73 \\
\hline Firmicutes & Phylum & 5.23 & 0.01 & 77.84 & 46.10 \\
\hline Proteobacteria & Phylum & 4.94 & 0.00 & 0.82 & 18.65 \\
\hline Gammaproteobacteria & Class & 4.92 & 0.00 & 0.76 & 17.64 \\
\hline Enterobacterales & Order & 4.68 & 0.00 & 0.42 & 9.82 \\
\hline Lactobacillus_uc & Species & 4.54 & 0.03 & 9.72 & 2.62 \\
\hline Coriobacteriales & Order & 4.51 & 0.01 & 0.76 & 6.94 \\
\hline Coriobacteriia & Class & 4.51 & 0.01 & 0.76 & 6.94 \\
\hline Coriobacteriaceae & Family & 4.51 & 0.01 & 0.76 & 6.94 \\
\hline Atopobium & Genus & 4.50 & 0.01 & 0.45 & 6.43 \\
\hline Pseudomonadales & Order & 4.45 & 0.01 & 0.31 & 6.38 \\
\hline Bacteroidetes & Phylum & 4.44 & 0.02 & 2.36 & 8.03 \\
\hline Atopobium vaginae & Species & 4.44 & 0.01 & 0.27 & 5.50 \\
\hline Pseudomonadaceae & Family & 4.43 & 0.03 & 0.29 & 6.13 \\
\hline Pseudomonas & Genus & 4.42 & 0.03 & 0.29 & 6.00 \\
\hline Prevotellaceae & Family & 4.38 & 0.05 & 2.24 & 7.31 \\
\hline Morganellaceae & Family & 4.37 & 0.00 & 0.00 & 4.38 \\
\hline Proteus & Genus & 4.36 & 0.01 & 0.00 & 4.26 \\
\hline Tissierellia & Class & 4.36 & 0.00 & 1.92 & 6.54 \\
\hline Tissierellales & Order & 4.36 & 0.00 & 1.92 & 6.54 \\
\hline Peptoniphilaceae & Family & 4.36 & 0.00 & 1.92 & 6.54 \\
\hline Prevotella & Genus & 4.34 & 0.05 & 2.22 & 6.87 \\
\hline Enterobacteriaceae & Family & 4.31 & 0.02 & 0.38 & 4.58 \\
\hline Proteus vulgaris group & Species & 4.31 & 0.01 & 0.00 & 3.83 \\
\hline Escherichia & Genus & 4.30 & 0.04 & 0.37 & 4.43 \\
\hline Escherichia coli group & Species & 4.26 & 0.04 & 0.33 & 4.03 \\
\hline Clostridia & Class & 4.08 & 0.01 & 0.46 & 2.98 \\
\hline Clostridiales & Order & 4.08 & 0.01 & 0.46 & 2.98 \\
\hline Bacillales & Order & 4.04 & 0.02 & 0.29 & 2.42 \\
\hline Lactobacillaceae_uc & Genus & 4.00 & 0.02 & 2.70 & 0.71 \\
\hline
\end{tabular}

Only those with $p<0.05$ and linear discriminant analysis (LDA) effect size $>4$ are presented.

$\%$ refers to the percentage of distribution occupied by each group.

PRE, pre-menopause group; POST, post-menopause group.

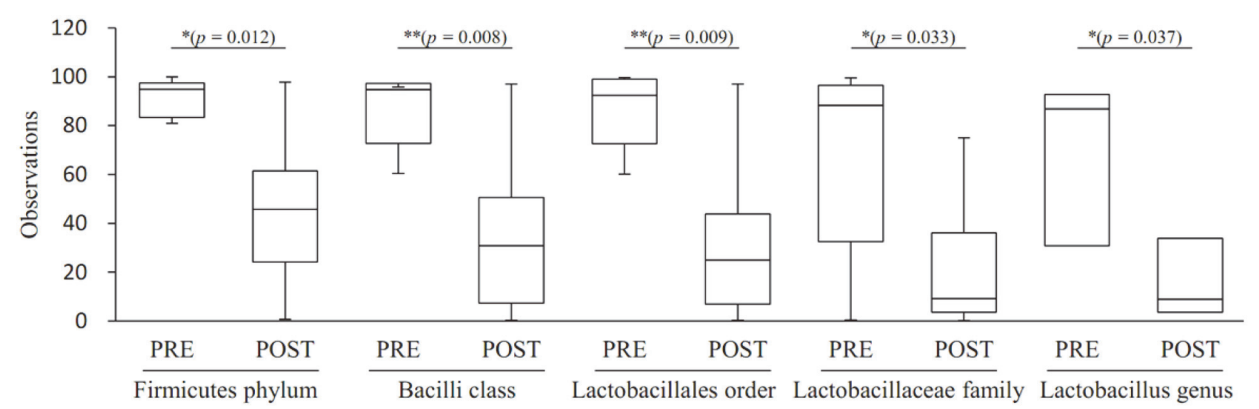

Fig. 7. Taxonomic abundance with an LDA effect size of more than 5. Taxonomic relative abundance was analyzed for the two groups using boxplots. The horizontal thick black band represents the median value, and the boxplot margins indicate the first and third quartiles. Statistical significance between groups was analyzed using the Wilcoxon rank-sum test ${ }^{*}$, $\left.p<0.05 ;{ }^{* *}, p<0.01\right)$.

\section{Vaginal Organic Acids by Metabolomics Analysis}

Fig. 8 shows the results of a quantitative analysis of organic acids in vaginal fluid. In the pre-menopausal group, lactate accounted for the highest percentage, $98.0 \%$ of the total organic-acid content. Post-menopausally, lactate concentration was $94.2 \%$, which was significantly lower $(p=0.015)$. The concentrations of pyruvate, 4 hydroxyphenyl acetate, 2-hydroxylovalerate, succinate, benzoate, isovalerate, butyrate, and malonate were lower than that of lactate, and there were no significant differences for any of these. 


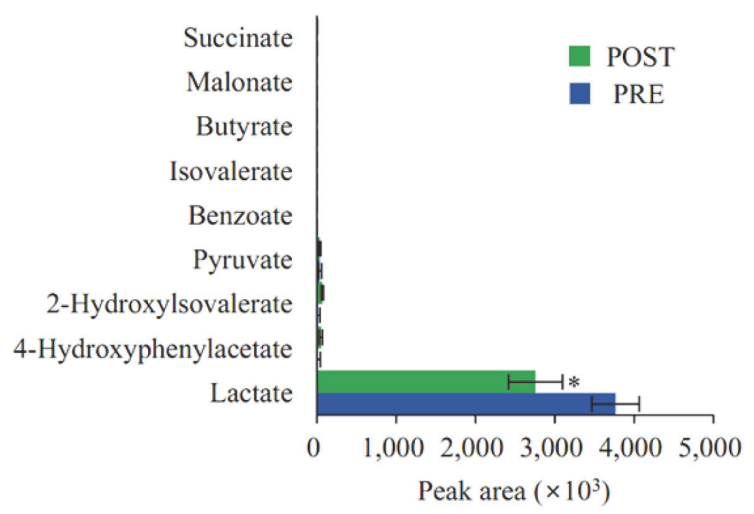

Fig. 8. The relative levels of organic acids in pre- and post-menopausal vaginal secretions. Data are presented as means with standard errors. ${ }^{*}, p<0.05$; PRE, pre-menopause group; POST, post-menopause group.

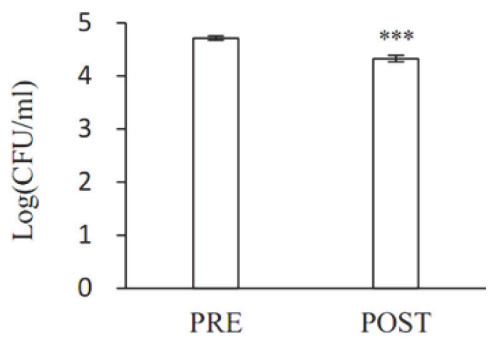

Fig. 9. Quantitative comparison of live Lactobacillus in vaginal fluid. Y-axis means CFU of Lactobacillus in the collected vaginal fluid. Values are expressed as mean and standard deviations. Statistical significance of the post-menopausal (POST) group compared to the pre-menopausal (PRE) group was analyzed using an unpaired Student's $t$-test. ${ }^{* * *}, p<0.001$.

\section{Quantification of Lactobacillus from Vaginal Fluid}

Lactobacillus in vaginal fluid samples collected was quantitatively analyzed through the culture method using MRS media (Fig. 9). Lactobacillus in vaginal fluid samples was $5.1 \times 10^{4} \mathrm{CFU} / \mathrm{ml}$ in pre-menopausal women and $2.1 \times 10^{4} \mathrm{CFU} / \mathrm{ml}$ in post-menopausal women. The amount of Lactobacillus in post-menopausal women confirmed by the culture method was $41.1 \%$ of the amount of Lactobacillus in pre-menopausal women, which was statistically significantly lower $\left({ }^{* *} p<0.001\right)$.

\section{Discussion}

About 20 years ago, the human microbiome was a fledgling field, but it is now thriving with investigations integrating basic and clinical sciences [41]. Microbiome studies of the digestive system have found associations between the human gut microbiome and cancer, neurodegenerative diseases, obesity, diabetes, and liver diseases; and by extension, the human gut microbiota is considered a potential source of new therapeutic agents [42]. Vaginal microbiome research has also been conducted over the past 10 years and has been found to be essential for maintaining vaginal health and host protection from disease and is related to behavior, health outcomes, race, ethnicity, and hygiene [43].

In this study, we performed a vaginal microbiome analysis on 11 pre-menopausal women and 19 postmenopausal women. There was a statistically significant difference in the composition of the taxonomic distribution in the two groups. Lactobacillus taxa dominated in pre-menopausal women, but our data confirmed that it decreased after menopause. In this survey, the Lactobacillus genus decreased from $63.2 \%$ pre-menopausal to $23.7 \%$ post-menopausal. This result is consistent with an earlier study in American women, which showed a reduction in the Lactobacillus genus from 83 to $54 \%$ post-menopause [44]. However, quantitatively, there was a difference between the two studies, and it seems that there is a need for studies in different ethnic groups.

Species abundance was lower, and species diversity was higher in post-menopausal women than in premenopausal women. This difference is probably due to the reduced abundance of Lactobacillus, which acts as an antimicrobial, and as a result, pathogens that previously colonized the vagina at a low rate have multiplied or become newly infectious. In a vaginal microbiome analysis of a female patient with pelvicitis in China, a decrease in lactobacilli was accompanied by polymicrobial infection, consistent with the current results [45].

LEfSe analysis showed that among all taxa corresponding to all taxon ranks, the decrease in the Lactobacillus taxa had the most considerable effect on the differences seen before and after menopause. In addition, metabolite analysis confirmed that lactic acid, produced mainly by Lactobacillus, was present at significantly lower concentrations in post-menopausal women. Lactobacillus can cause vaginal eubiosis by killing dysbiotic microbes 
and various pathogens through lactic acid production, a primary antibacterial substance [46]. From these results, we would predict that Lactobacillus may be used to alleviate diseases related to menopause. A clinical trial in Korea reported that oral ingestion of lactobacilli by post-menopausal women improved symptoms related to menopause [47], but further studies are needed to investigate alleviation of menopause-related symptoms by administering Lactobacillus directly to the vagina rather than by oral administration to restore the vaginal microbiome to a premenopausal state.

In addition to menopause, the vaginal microbiome is associated with vaginal infections, such as pelvic vaginitis, bacterial vaginosis, vulvar candidiasis, sexually transmitted diseases, and human immunodeficiency virus (HIV) infection $[48,49]$. Also, changes in the vaginal microflora can lead to severe gynecological problems, such as pregnancy loss, premature birth, and low pregnancy rates [50]. Therefore, the results of this study will contribute to broadening the understanding of not only menopause but also various gynecological diseases, and further studies will be needed for this. However, it is considered as a limitation of this study that the study subjects are not sufficient. In addition, more in-depth studies and analyses of the microbiome according to menopause by age group are needed.

\section{Acknowledgments}

This research was financially supported by the Ministry of Trade, Industry, and Energy (MOTIE), Korea, under the "Regional Industry-based Organization Support Program" (Ref. No. P0001942) supervised by the Korean Institute for Advancement of Technology (KIAT). This study was also supported by the Soonchunhyang University Research Fund.

\section{Conflict of Interest}

The authors have no financial conflicts of interest to declare.

\section{Literature}

1. Marchesi JR, Ravel J. 2015. The vocabulary of microbiome research: a proposal. Microbiome 3: 31 .

2. Human Microbiome Project C. 2012. A framework for human microbiome research. Nature 486: 215-221.

3. Sender R, Fuchs S, Milo R. 2016. Revised estimates for the number of human and bacteria cells in the body. PLoS Biol. 14: e1002533.

4. Grice EA, Segre JA. 2012. The human microbiome: our second genome. Annu. Rev. Genom. Hum. G. 13: 151-170.

5. Zhao LP. 2010. GENOMICS The tale of our other genome. Nature 465: 879-880.

6. Qin JJ, Li RQ, Raes J, Arumugam M, Burgdorf KS, Manichanh C, et al. 2010. A human gut microbial gene catalogue established by metagenomic sequencing. Nature 464: 59-U70.

7. Lloyd-Price J, Abu-Ali G, Huttenhower C. 2016. The healthy human microbiome. Genome Medicine 8.

8. Ma B, Forney LJ, Ravel J. 2012. Vaginal microbiome: rethinking health and disease. Annu. Rev. Microbiol. 66: 371-389.

9. Gupta S, Kakkar V, Bhushan I. 2019. Crosstalk between vaginal microbiome and female health: A review. Microb. Pathog. 136

10. Fettweis JM, Serrano MG, Sheth NU, Mayer CM, Glascock AL, Brooks JP, et al. 2012. Species-level classification of the vaginal microbiome. BMC Genomics. 13 Suppl 8: S17.

11. Ravel J, Gajer P, Abdo Z, Schneider GM, Koenig SS, McCulle SL, et al. 2011. Vaginal microbiome of reproductive-age women. Proc. Natl. Acad. Sci. USA 108 Suppl 1: 4680-4687.

12. Srinivasan S, Hoffman NG, Morgan MT, Matsen FA, Fiedler TL, Hall RW, et al. 2012. Bacterial communities in women with bacterial vaginosis: high resolution phylogenetic analyses reveal relationships of microbiota to clinical criteria. PLoS One 7: e37818.

13. Wang Y, Zhang Y, Zhang Q, Chen H, Feng Y. 2018. Characterization of pelvic and cervical microbiotas from patients with pelvic inflammatory disease. J. Med. Microbiol. 67: 1519-1526.

14. MacIntyre DA, Chandiramani M, Lee YS, Kindinger L, Smith A, Angelopoulos N, et al. 2015. The vaginal microbiome during pregnancy and the postpartum period in a European population. Sci. Rep. 5: 8988.

15. Fettweis JM, Brooks JP, Serrano MG, Sheth NU, Girerd PH, Edwards DJ, et al. 2014. Differences in vaginal microbiome in African American women versus women of European ancestry. Microbiology 160: 2272-2282.

16. Takahashi TA, Johnson KM. 2015. Menopause. Med. Clin. N Am. 99: 521-534.

17. Nelson HD. 2008. Menopause. Lancet. 371: 760-770.

18. Fait T. 2019. Menopause hormone therapy: latest developments and clinical practice. Drugs Context. 8: 212551.

19. Gustafsson RJ, Ahrne S, Jeppsson B, Benoni C, Olsson C, Stjernquist M, et al. 2011. The Lactobacillus flora in vagina and rectum of fertile and postmenopausal healthy Swedish women. BMC Womens Health 11: 17.

20. Zhang R, Daroczy K, Xiao B, Yu L, Chen R, Liao Q. 2012. Qualitative and semiquantitative analysis of Lactobacillus species in the vaginas of healthy fertile and postmenopausal Chinese women. J. Med. Microbiol. 61: 729-739.

21. Pabich WL, Fihn SD, Stamm WE, Scholes D, Boyko EJ, Gupta K. 2003. Prevalence and determinants of vaginal flora alterations in postmenopausal women. J. Infect. Dis. 188: 1054-1058.

22. Bolger AM, Lohse M, Usadel B. 2014. Trimmomatic: a flexible trimmer for Illumina sequence data. Bioinformatics 30: $2114-2120$.

23. Myers EW, Miller W. 1988. Optimal alignments in linear space. Comput. Appl. Biosci. 4: 11-17.

24. Wheeler TJ, Eddy SR. 2013. nhmmer: DNA homology search with profile HMMs. Bioinformatics 29: 2487-2489.

25. Rognes T, Flouri T, Nichols B, Quince C, Mahe F. 2016. VSEARCH: a versatile open source tool for metagenomics. PeerJ. 4: e2584.

26. Yoon SH, Ha SM, Kwon S, Lim J, Kim Y, Seo H, et al. 2017. Introducing EzBioCloud: a taxonomically united database of $16 \mathrm{~S}$ rRNA gene sequences and whole-genome assemblies. Int. J. Syst. Evol. Microbiol. 67: 1613-1617.

27. Chao A, Lee SM. 1992. Estimating the number of classes via sample coverage. J. Am. Stat. Assoc. 87: 210-217.

28. Chao A. 1987. Estimating the population-size for capture recapture data with unequal catchability. Biometrics 43: $783-791$.

29. Burnham KP, Overton WS. 1979. Robust estimation of population-size when capture probabilities vary among animals. Ecology 60: $927-936$.

30. Magurran AE. 2013. Measuring Biological Diversity. Chapter 4 - A index of diversity, "Nonparametric" measures of diversity, pp. 106-109, Wiley-Blackwell|.

31. Chao A, Shen TJ. 2003. Nonparametric estimation of Shannon's index of diversity when there are unseen species in sample. Environ. Ecol. Stat. 10: 429-443.

32. Faith DP. 1992. Conservation evaluation and phylogenetic diversity. Biol. Conserv. 61: 1-10.

33. Lin JH. 1991. Divergence measures based on the shannon entropy. Ieee T Inform. Theory. 37: 145-151. 
34. Beals EW. 1984. Bray-curtis ordination - An effective strategy for analysis of multivariate ecological data. Adv. Ecol. Res. 14: 1-55.

35. Chen J, Bittinger K, Charlson ES, Hoffmann C, Lewis J, Wu GD, et al. 2012. Associating microbiome composition with environmental covariates using generalized UniFrac distances. Bioinformatics 28: 2106-2113.

36. Hamady M, Lozupone C, Knight R. 2010. Fast UniFrac: facilitating high-throughput phylogenetic analyses of microbial communities including analysis of pyrosequencing and PhyloChip data. ISME J. 4: 17-27.

37. Segata N, Izard J, Waldron L, Gevers D, Miropolsky L, Garrett WS, et al. 2011. Metagenomic biomarker discovery and explanation. Genome Biol. 12: R60.

38. Wallis WHKaWA. 1952. Use of ranks in one-criterion variance analysis. J. Am. Stat. Assoc. 47: 583-621.

39. Yuan M, Breitkopf SB, Yang X, Asara JM. 2012. A positive/negative ion-switching, targeted mass spectrometry-based metabolomics platform for bodily fluids, cells, and fresh and fixed tissue. Nat. Protoc. 7: 872-881.

40. Vasquez A, Jakobsson T, Ahrne S, Forsum U, Molin G. 2002. Vaginal lactobacillus flora of healthy Swedish women. J. Clin. Microbiol. 40: 2746-2749.

41. 2019. After the Integrative Human Microbiome Project, what's next for the microbiome community? Nature 569: 599

42. Cani PD. 2018. Human gut microbiome: hopes, threats and promises. Gut. 67: 1716-1725.

43. Lewis FMT, Bernstein KT, Aral SO. 2017. Vaginal microbiome and its relationship to behavior, sexual health, and sexually transmitted diseases. Obstet. Gynecol. 129: 643-654.

44. Brotman RM, Shardell MD, Gajer P, Fadrosh D, Chang K, Silver MI, et al. 2014. Association between the vaginal microbiota, menopause status, and signs of vulvovaginal atrophy. Menopause 21: 450-458.

45. Wang Y, Zhang Y, Zhang Q, Chen H, Feng Y. 2018. Characterization of pelvic and cervical microbiotas from patients with pelvic inflammatory disease. J. Medi. Microbiol. 67: 1519-1526.

46. Tachedjian G, Aldunate M, Bradshaw CS, Cone RA. 2017. The role of lactic acid production by probiotic Lactobacillus species in vaginal health. Res. Microbiol. 168: 782-792.

47. Lim EY, Lee SY, Shin HS, Lee J, Nam YD, Lee DO, et al. 2020. The Effect of Lactobacillus acidophilus YT1 (MENOLACTO) on improving menopausal symptoms: a randomized, double-blinded, placebo-controlled clinical trial. J. Clin. Med. 9: 2173.

48. van de Wijgert J. 2017. The vaginal microbiome and sexually transmitted infections are interlinked: consequences for treatment and prevention. PLoS Med. 14: e1002478.

49. Eastment MC, McClelland RS. 2018. Vaginal microbiota and susceptibility to HIV. AIDS 32: 687-698.

50. Bracewell-Milnes T, Saso S, Nikolaou D, Norman-Taylor J, Johnson M, Thum MY. 2018. Investigating the effect of an abnormal cervico-vaginal and endometrial microbiome on assisted reproductive technologies: A systematic review. Am. J. Reprod. Immunol. 80: e13037. 